\title{
Clinical effect of a temporary pacemaker on an electrical storm induced by severe acute aconitine poisoning
}

\author{
FENG CHENG ${ }^{1}$, LI HAO $^{2}$ and MING-WEI LIU ${ }^{3}$ \\ Departments of ${ }^{1}$ Cardiology, and ${ }^{2}$ Emergency Medicine, Yan'an Hospital of Kunming City, Kunming, \\ Yunnan 650051; ${ }^{3}$ Department of Emergency Medicine, The First Hospital Affiliated to \\ Kunming Medical University, Kunming, Yunnan 650032, P.R. China
}

Received September 26, 2021; Accepted January 20, 2022

DOI: $10.3892 /$ wasj.2022.142

\begin{abstract}
In China, medicinal liquor made from the aconitine root soaked in wine is poisoned. At present, reliable detoxification drugs are not available. The present study used a temporary pacemaker to treat 12 patients who required treatment due to an electrical storm caused by aconitine poisoning between June, 2017 and April, 2021. The results revealed that temporary pacing was successful in all 12 patients. The electrode placement rate was $100 \%$, and the effective cardiac pacing rate was $100 \%$. The blood pressure of the patients gradually returned to normal levels. Ventricular tachycardia and high-grade atrioventricular block were terminated. As regards atrial premature beats and ventricular premature beats, the sinus rhythm was restored after continuous amiodarone pumping. Simultaneously, fluid supplementation and control supportive treatment were administered. The patients were hospitalized for 4-6 days. The patients' symptoms disappeared, their vital signs were stable and their electrocardiogram (ECG) exhibited normal findings. The 12 patients were followed-up for 1 week following discharge and did not have any discomfort; the ECG revealed no abnormalities. On the whole, the findings of the present study suggest that temporary cardiac pacing may be an effective treatment for malignant arrhythmias caused by severe acute aconitine poisoning. However, the present study only involved a very small number of cases. In the future, further studies are required with larger sample, and further randomized, controlled multi-center studies are also warranted to these findings.
\end{abstract}

Correspondence to: Professor Li Hao, Department of Emergency Medicine, Yan'an Hospital of Kunming City, 245 Renmin East Road, Panlong, Kunming, Yunnan 650051, P.R. China

E-mail: 807323152@qq.com

Professor Ming-Wei Liu, Department of Emergency Medicine, The First Hospital Affiliated to Kunming Medical University, 295 Xichang Road, Wu Hua, Kunming, Yunnan 650032, P.R. China E-mail:1mw2004210@163.com

Keywords: temporary pacemaker, aconitine poisoning, electric wind burst, treatment

\section{Introduction}

Aconitine exerts pharmacological effects, such as dispelling wind and dampness, and easing menstrual cramps and pain (1). In China, it is considered common practice to soak Radix aconitiagrestis and Radix aconiti sinensisin wine or to add Kusnezoff monkshood root slices when cooking food. However, the majority of plants of the genus Aconitum are poisonous. Aconitine is the main toxic component of Aconitum plants. It exerts effects mainly on the heart and nervous system, causing a variety of arrhythmias and leading to death (1). Its therapeutic dose is close to the poisonous or lethal dose, and it is a typical representative of poisonous plants with medicinal value (2). The oral administration of $0.2 \mathrm{mg}$ aconitine can be poisonous, and a dose of 3-5 $\mathrm{mg}$ can cause death. During use, aconitine can easily cause poisoning and death due to individual differences in drug tolerance, improper usage or misuse and deliberate poisoning (3). The cause of death is mostly malignant arrhythmia, and the hospital mortality rate in China is $5.5 \%$ (3). In China, medicinal liquor made from aconitine root soaked in wine is mostly poisoned.

At present, reliable detoxification drugs are not available. In addition to inducing vomiting, gastric lavage and catharsis, the key to treating aconitine poisoning is to correct arrhythmia in a timely and effective manner, stabilize myocardial cells, and maintain the stability of vital signs (4). The majority of patients are treated with atropine, lidocaine, or amiodarone, which can effectively control arrhythmia and cure patients. However, some severely ill patients have severe arrhythmia, such as an electrical storm, and thus do not survive due to ineffective treatment with antiarrhythmic drugs. Temporary pacemakers enable pacing and biphasic defibrillation, which can pace the heart in a short period of time, and also defibrillate, restore autonomous heart rhythm, prevent malignant arrhythmia, and ensure blood supply to vital organs (5). To the best of our knowledge, no study to date has used temporary pacemakers for the treatment of malignant arrhythmias induced by aconitine poisoning. Therefore, the present study used a temporary pacemaker to treat 12 patients who were admitted to the Department of Emergency Medicine of Yan'an Hospital in Kunming from June, 2017 to April, 2021 due to an electrical storm caused by aconitine poisoning, and all were cured. 


\section{Patients and methods}

Patient information. From June, 2017 to April, 2021, 12 patients were admitted to the Department of Emergency Medicine of Yan'an Hospital in Kunming due to aconitine poisoning. Among them, 8 patients were males, and 4 patients were females. The patients' age ranged from 33 to 65 years, with an average age of 45 years. Poisoning was induced in 2 patients due to the ingestion of herbal black powder, and in 10 patients due to the consumption of medicated wine. Symptoms occurred after 10-30 min, and they all visited the Department of Emergency Medicine within 1-2 $\mathrm{h}$ following the onset of symptoms. The present study was approved by the First Affiliated Hospital of Kunming Medical University Research Ethics Boards. Written informed consent for participation in the study was obtained from the parent or relative of the participants.

Inclusion criteria. The inclusion criteria were as follows: i) All the 12 patients met the diagnostic criteria for ventricular tachycardia electrical storm: Patients who suffered from two or more spontaneous ventricular tachycardias or ventricular fibrillation within $24 \mathrm{~h}$, sharp and severe disturbance of cardiac electrical activity, rapid ventricular tachycardia, and ventricular fibrillation that can occur repeatedly requiring repeated electrical cardioversion; ii)treatment with atropine, lidocaine, or amiodarone was ineffective; iii) electrical cardioversion therapy was ineffective; iv) suffered from combined high-grade atrioventricular block; v) suffered from QT syndrome with torsades de pointes; vi) patients and relatives agreed to the use of temporary pacemakers.

Exclusion criteria. The exclusion criteria were as follows: i) Patients in which a ventricular tachycardia electrical storm was caused by non-aconitine poisoning; ii) treatment with atropine, lidocaine, or amiodarone was effective; iii) electrical cardioversion was effective; iv) patients and relatives did not agree to the use of temporary pacemakers.

Clinical manifestations. All 12 cases exhibited symptoms, such as numbness in the mouth, tongue, face and limbs, and other symptoms including nausea, vomiting, dizziness, sweating, chest tightness and palpitations. Among the patients, there were two cases of convulsions with unconsciousness during medical treatment, six cases of syncope, two cases of convulsions with facial cyanosis, and two cases of whole-body numbness and a 'feeling of dying' (Table I).

Electrocardiogram (ECG). The first ECG or ECG monitoring revealed five cases of multi-source ventricular premature beats, six cases of polymorphic ventricular tachycardia, four cases of ventricular fibrillation, and three cases of torsades de pointes ventricular tachycardia. All 12 cases had varying degrees of hypotension (Table I). A typical ECG is illustrated in Fig. 1.

Treatment method. The 12 patients were immediately administered a thorough gastric lavage, activated charcoal plus $250 \mathrm{ml}$ of mannitol oral catharsis, continuous ECG monitoring, oxygen inhalation, routine fluid replacement, intravenous supplementation of potassium and magnesium polarized fluids, correction of acidosis and electrolyte disorders, and
$102 \mathrm{mg}$ of intravenous atropine. Furosemide (Shaanxi Jingxi Pharmaceutical Co., Ltd.; $20 \mathrm{mg}$ ) is an intravenous diuretic. Lidocaine (Zhejiang Chengxin Pharmaceutical Co., Ltd.; $100 \mathrm{mg}$ ) was injected intravenously every $15-30 \mathrm{~min}$. Amiodarone (Shandong Fangming Pharmaceutical Group Co., Ltd.) was used when lidocaine (Zhejiang Chengxin Pharmaceutical Co., Ltd.) was ineffective. All patients were administered a slow intravenous bolus of $150 \mathrm{mg}$ of amiodarone (Shandong Fangming Pharmaceutical Group Co., Ltd.), followed by continuous micropump pumping of $1 \mathrm{mg} / \mathrm{min}$ amiodarone (Shandong Fangming Pharmaceutical Group Co., Ltd.). The aforementioned 'rescue' methods were not effectivein 10 patients, who manifested a loss of consciousness, shock, ventricular fibrillation and Adams-Stokes syndrome. The patients were immediately administered electric shock defibrillation, chest compressions and emergency bedside temporary cardiac pacing for rescue.

Equipment. The equipment used included a Medtronic Temporary Pacemaker T10 (Shanghai Zanzhuo Medical Instrument Co., Ltd.), no. 18 thin-walled puncture needle and J-shaped guide wire (Shenzhen Yixinda Medical New Technology Co., Ltd.), 6F bipolar electrode catheter (Beijing Wandong Medical Equipment Co., Ltd.), 6F venous dilator and venous sheath (Beijing Wandong Medical Equipment Co., Ltd.), a photoelectric electrocardiograph and a Minadray 9000 ECG monitor (Nanjing Baden Medical Co., Ltd.).

Operation method. For the procedure, the patient was placed on his/her back and monitored using an ECG. Following local infiltration anesthesia, the left subclavian vein was punctured by Seldinger's method, and the pacing electrode was successfully inserted. Bedside ECG monitoring was used for temporary cardiac pacing. After the puncture was successful, the outer sheath was rapidly fed into the $6 \mathrm{~F}$ electrode catheter with a slightly curved tip end, and the electrode catheter was fed $20-25 \mathrm{~cm}$. A temporary pacemaker was connected. The pacemaker voltage and sensitivity were adjusted to 3 and $0.8 \mathrm{mV}$, respectively. The pacing frequency was 60 or 10 beats/min higher than the patient's own frequency. Once the main wave in leads II, III and aVF appeared downward, and lead V1 presented a pacing pattern with left bundle branch block wide malformation, the ECG was traced. The pacing threshold $<1.0 \mathrm{~V}$ was determined, and the lead was fixed and placed locally. Finally, the operation was complete.

\section{Results}

Effect of temporary pacemaker on wind burst induced by severe acute aconitine poisoning. Temporary pacing was successful in all 12 patients. The electrode placement rate was $100 \%$, and the effective cardiac pacing rate was $100 \%$. The patients' blood pressure gradually returned to normal. Ventricular tachycardia and high-grade atrioventricular block were terminated. For atrial premature beats and ventricular premature beats, the sinus rhythm was restored following continuous amiodarone pumping (Fig. 2). At the same time, fluid supplementation and control supportive treatment were administered. The patients were hospitalized for 4-6 days. The patients' symptoms disappeared, their vital signs were stable 


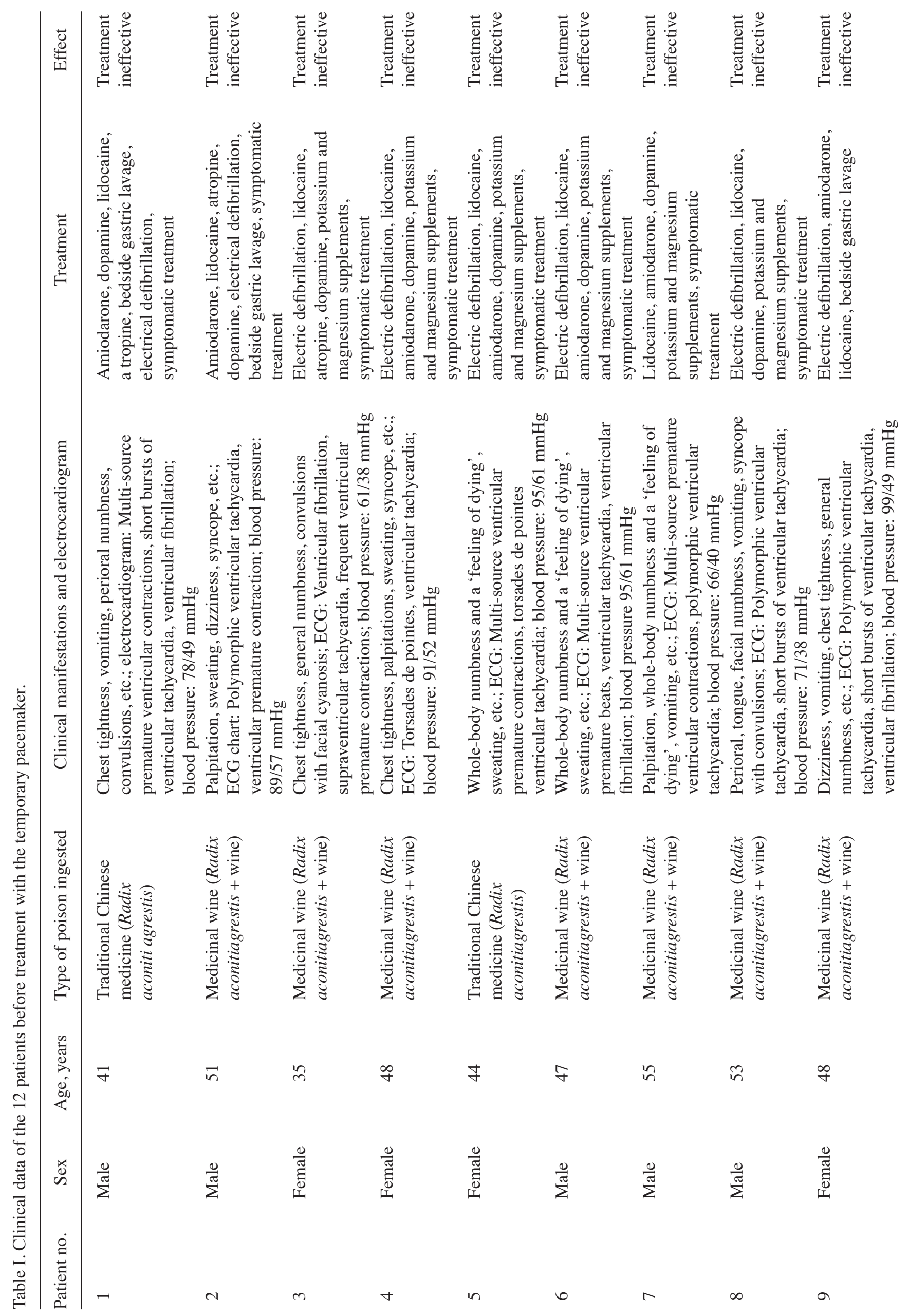




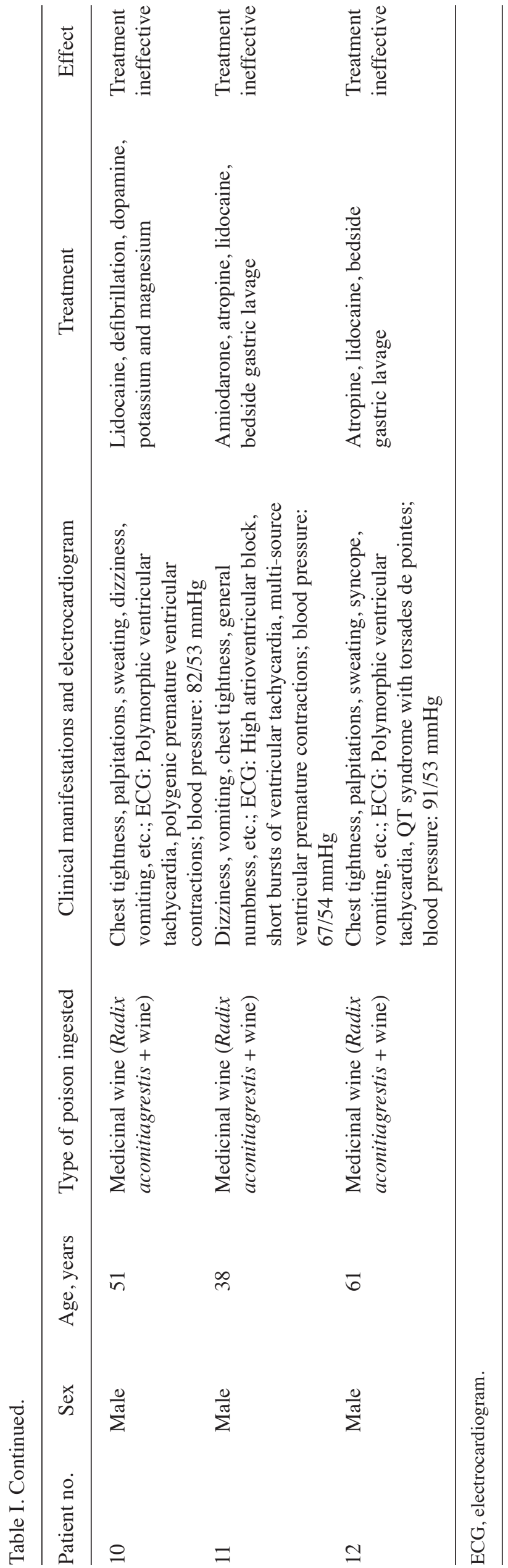

and their ECG exhibited normal findings. No abnormalities in the ECG were observed for 2 days, and the patients were discharged for follow-up.

Side-effects of the temporary pacemaker. In total, 1 patient experienced pacing dysfunction caused by temporary pacemaker displacement. Pacemaker malfunction was corrected after adjusting the position of the pacemaker.

Patient follow-up. The 12 patients were followed-up for 1 week following discharge without any discomfort, and the ECG did not reveal any abnormalities (Fig. 3).

\section{Discussion}

Aconitine mainly damages the circulatory and central nervous systems. The effects of aconitine poisoning on the heart excite the vagus nerve, causing the postganglionic fibers to release a large amount of acetylcholine, thereby reducing the autonomy and conductivity of the sinus node. Aconitine also has a direct effect on the myocardium, which renders the excitatory conduction and refractory period of each part of the myocardium inconsistent. Moreover, repolarization is not synchronized to form re-entry, resulting in severe ventricular arrhythmia. Ventricular tachycardia is the most common and likely cause of death. Aconitine can directly act on ventricular muscles, producing high-frequency ectopic rhythms and causing ventricular fibrillation, cardiogenic shock and Adams-Stokes syndrome (6). Therefore, malignant arrhythmia induced by acute aconitine poisoning can be life-threatening if not corrected in a timely manner.

Temporary cardiac pacemakers have biphasic defibrillation and pacing functions and play an important role in rescuing patients undergoing cardiac arrest and bradyarrhythmia (7). It has been shown that the defibrillation rate of temporary cardiac pacing can reach $100 \%$, and the pacing success rate of patients with bradycardia is $>80 \%$ (8). In addition, the energy required for biphasic defibrillation is minimal, the damage to the patient's myocardium is minor, and the time it takes for the heart to restore sinus rhythm after defibrillation is short. Temporary cardiac pacing therapy can stabilize the patients' hemodynamics and reduce the recurrence rate of cardiac arrest $(9,10)$. In the present study, 12 patients were administered temporary pacemaker therapy, which controlled ventricular tachycardia and electrical storms in a timely manner, and maintained the vital signs of the patients. In addition to the temporary pacemaker, fluid replacement and antiarrhythmic treatment with amiodarone were continued. All 12 patients were cured.

The more common complications of temporary cardiac pacing include pacing disorder, arrhythmia, myocardial perforation, pericardial tamponade, pneumothorax, infection and puncture complications (such as subcutaneous hematoma and venous thrombosis) (11-13). In the present study, 1 patient experienced pacing dysfunction caused by temporary pacemaker displacement. Pacemaker malfunction was corrected after adjusting the position of the pacemaker.

Therefore, temporary cardiac pacing may be an effective treatment for malignant arrhythmias caused by severe acute aconitine poisoning. However, the present study has some limitations which are as follows: The present study involved 

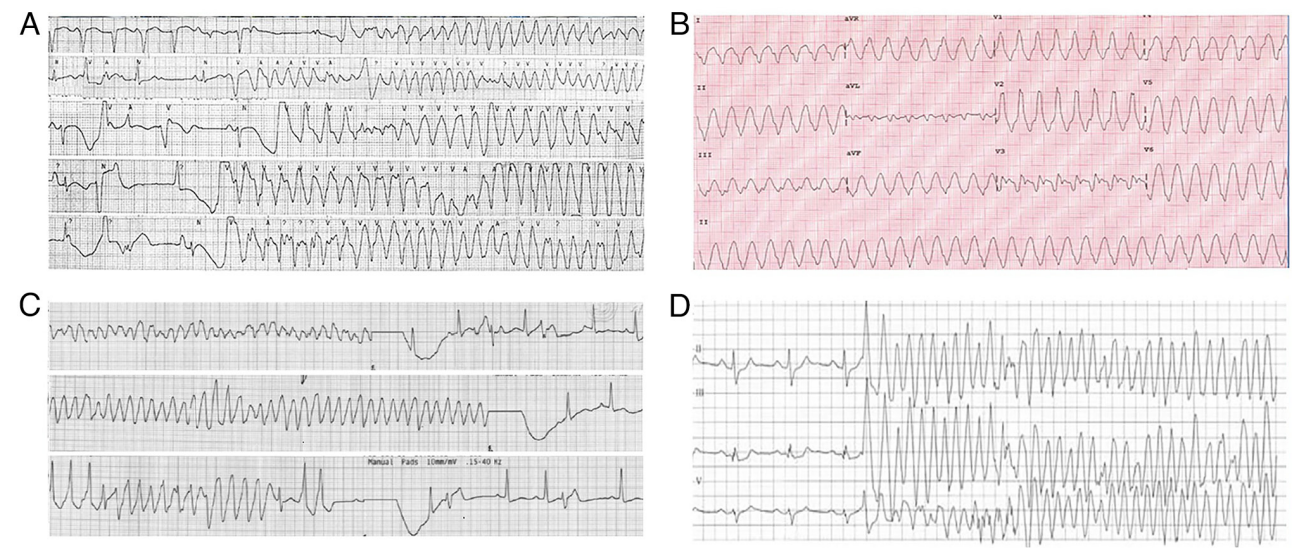

Figure 1. Typical ECG before treatment with a temporary pacemaker from different patients. (A-D) Four different ECGs are shown. (A) Sinus rhythm, torsades de pointes tachycardia. (B) Sinus rhythm, ventricular tachycardia. (C) Sinus rhythm, torsade de pointes tachycardia. (D) Sinus rhythm, torsade de pointes tachycardia. ECG, electrocardiogram.

A

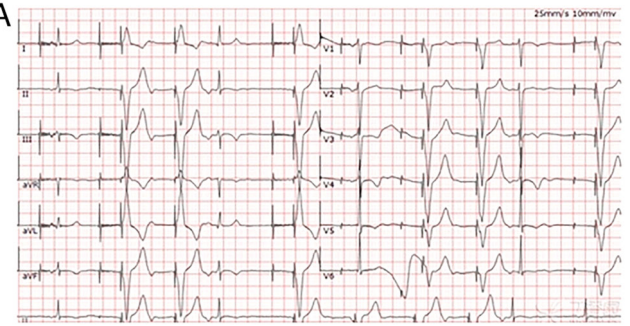

$\mathrm{C}$

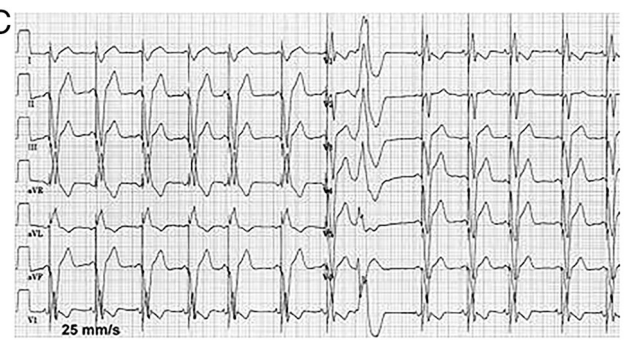

B

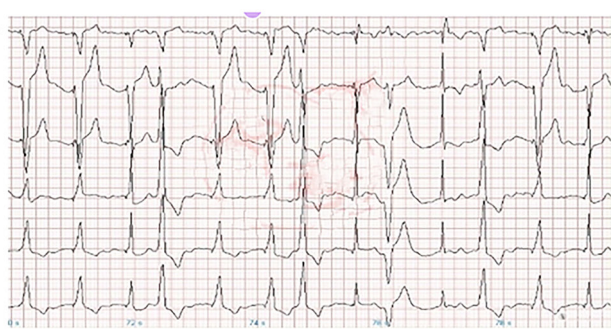

$\mathrm{D}$

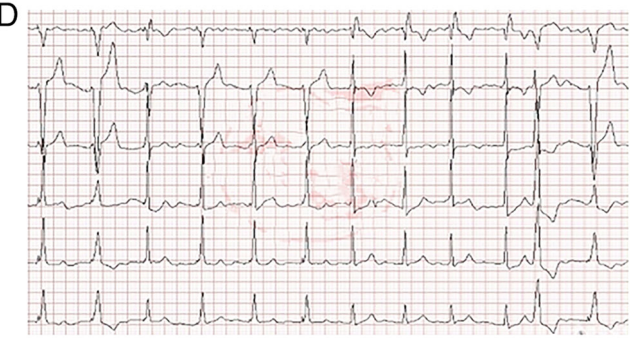

Figure 2. Typical ECG following treatment with a temporary pacemaker from different patients. (A-D) Four different ECGs are shown. (A) Paced rhythm. (B) Pacing rhythm, frequent premature ventricular contractions. (C) Paced rhythm, premature ventricular contractions. (D) Paced rhythm, premature ventricular contractions. ECG, electrocardiogram.

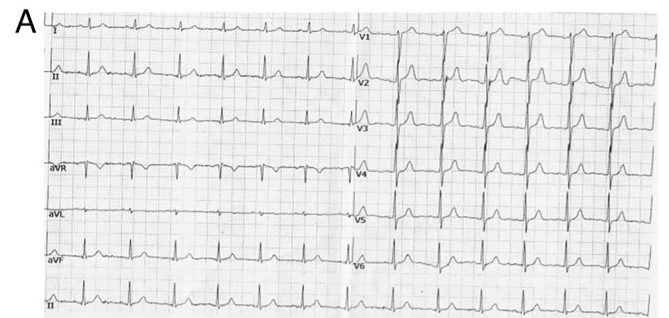

C

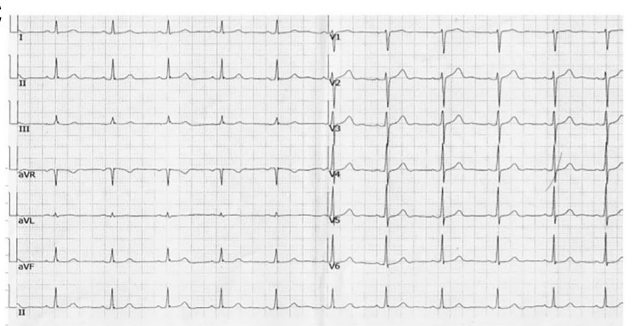

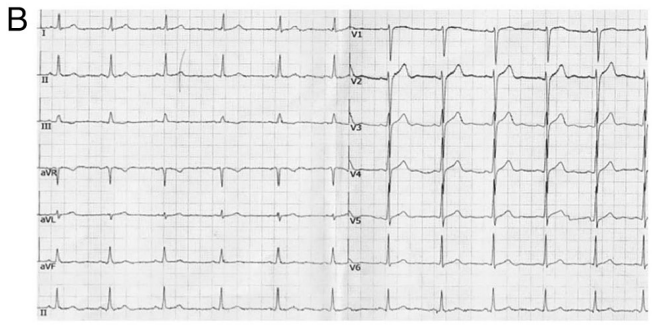

$\mathrm{D}$

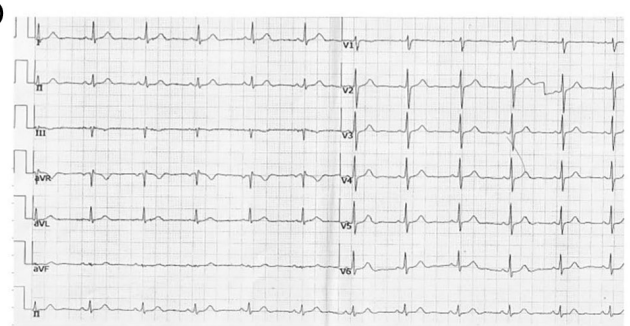

Figure 3. Typical ECG at follow-up from different patients at 1 week following discharge. (A-D) Four different ECGs are shown. (A) Sinus rhythm, normal electrocardiogram. (B) Sinus rhythm, normal electrocardiogram. (C) Sinus rhythm, normal electrocardiogram. (D) Sinus rhythm, normal electrocardiogram. ECG, electrocardiogram. 
a very small number of cases, and the data provided are thus limited. Therefore, further studies with larger sample sizes are required, and farther randomized, controlled multi-center studies are also warranted to confirm the current findings. There are reports on the implantation of a temporary external transvenous implantable cardioverter-defibrillator as a bridge for reimplantation following infected device extraction $(14,15)$. Those temporarily implanted devices with a dual-coil ICD lead can deliver shocks (16); however, they were not used in the present study. A temporary pacemaker cannot treat ventricular tachycardia/ventricular fibrillation with biphasic cardioversion or defibrillation. Advanced life support/advanced cardiac life support for hemodynamically unstable patients with malignant ventricular arrhythmias is always electrical cardioversion, or CPR and defibrillation in case of cardiac arrest. Temporary pacemakers may be useful for maintaining stable cardiac output after effective defibrillation/cardioversion or they can provide overdrive pacing that can terminate fast ventricular rhythms. However, they are not a treatment of choice for patients with an electrical storm where external defibrillation/cardioversion is a priority. A more in-depth analysis of the side-effects which may occur prior to large-scale implementation is thus essential.

\section{Acknowledgements}

Not applicable.

\section{Funding}

The present study received funding via a grant provided by the National Natural Science Foundation (grant no. 81960350).

\section{Availability of data and materials}

The datasets used and/or analyzed during the current study are available from the corresponding author on reasonable request.

\section{Authors' contributions}

FC, LH and MWL made contributions to the acquisition and analysis of the data. FC and MWL contributed to the interpretation of the data. FC, LH and MWL made contributions to the conception and design of the study and drafted the manuscript. LH and MWL prepared Figs. 1-3. FC, LH and MWL confirm the authenticity of all the raw data. All authors have read and approved the final manuscript.

\section{Ethics approval and consent to participate}

The present study was approved by the First Affiliated Hospital of Kunming Medical University Research Ethics Boards. Written informed consent for participation in the study was obtained from the parent or relative of participants.

\section{Patient consent for publication}

Consent for patient data to be published was provided by the patients themselves or their relatives.

\section{Competing interests}

The authors declare that they have no competing interests.

\section{References}

1. Tomlinson B, Chan TY, Chan JC and Critchley JA: Herb-induced aconitine poisoning. Lancet 341: 370-371, 1993.

2. Jeon SY, Jeong W, Park JS, You Y, Ahn HJ, Kim S, Kim D, Park D, Chang H and Kim SW: Clinical relationship between blood concentration and clinical symptoms in aconitine intoxication. Am J Emerg Med 40: 184-187, 2021.

3. Jin R, Wang YG and Zhang B: Establishment and verification of risk assessment scale for clinical safety medication of aconitine. Zhongguo Zhong Yao Za Zhi 43: 222-226, 2018 (In Chinese).

4. Cho YS, Choi HW, Chun BJ, Moon JM and Na JY: Quantitative analysis of aconitine in body fluids in a case of aconitine poisoning. Forensic Sci Med Pathol 16: 330-334, 2020.

5. Chatzidou S, Kontogiannis C, Tsilimigras DI, Georgiopoulos G, Kosmopoulos M, Papadopoulou E, Vasilopoulos G and Rokas S: Propranolol versus metoprolol for treatment of electrical storm in patients with implantable cardioverter-defibrillator. J Am Coll Cardiol 71: 1897-1906, 2018.

6. Pan MC, Zhou XW, Liu Y, Wang YN, Qiu XG, Wu SF and Liu Q: Research progress on the molecular mechanisms of toxicology of ethanol-aconitine induced arrhythmia. Fa Yi Xue Za Zhi 36: 115-119, 2020 (In English, Chinese).

7. He WW and Chu YJ: Clinical investigation of temporary heart pacemaker for emergency treatment of patients with acute and severe cardiovascular diseases. Zhonghua Yi Xue Za Zhi 96: 2644-2647, 2016 (In Chinese).

8. Sert A, Aypar E, Odabas D and Aygul MU: Temporary cardiac pacemaker in the treatment of junctional rhythm and hypotension due to imipramine intoxication. Pediatr Cardiol 32: 521-524, 2011.

9. Lee MS, Nguyen H and Shlofmitz R: Incidence of bradycardia and outcomes of patients who underwent orbital atherecto-my without a temporary pacemaker. J Invasive Cardiol 29: 59-62, 2017.

10. Kowey PR, Mullan DF and Wetstein L: Pacemaker therapy. Surg Clin North Am 65: 595-611, 1985.

11. Metkus TS, Schulman SP, Marine JE and Eid SM: Complications and outcomes of temporary transvenous pacing: An analysis of $>$ 360,000 patients from the national inpatient sample. Chest 155: 749-757, 2019.

12. Zhou X, Ze F, Li D, Li X and Wang B: Outcomes of temporary pacing using active fixation leads and externalized permanent pacemakers in patients with cardiovascular implantable electronic device infection and pacemaker dependency. J Cardiovasc Electrophysiol 32: 3051-3056, 2021.

13. Buttigieg J, Asciak R and Azzopardi CM: Pacemaker lead-associated thrombosis in cardiac resynchronisation therapy. BMJ Case Rep 2015: bcr2015210314, 2015.

14. Dębski M, Ząbek A, Boczar K, Urbańczyk-Zawadzka M, Lelakowski J and Małecka B: Temporary external implantable cardioverter-defibrillator as a bridge to reimplantation after infected device extraction. J Arrhythm 34: 77-80, 2017.

15. Da Rosa MR, Sapp JL, Howlett JG, Falkenham A and Légaré JF: Implantable cardioverter-defibrillator implantation as a bridge to cardiac transplantation. J Heart Lung Transpl 26: 1336-1339, 2007.

16. Kumar P, Baker M and Gehi AK: Comparison of single-coil and dual-coil implantable defibrillators: A meta-analysis. JACC Clin Electrophysiol 3: 12-19, 2017. 